\title{
Polarity Control in Group-III Nitrides beyond Pragmatism
}

\author{
Stefan Mohn, ${ }^{1, *}$ Natalia Stolyarchuk, ${ }^{1,2}$ Toni Markurt, ${ }^{1}$ Ronny Kirste, ${ }^{3}$ Marc P. Hoffmann, ${ }^{3}$ Ramón Collazo, ${ }^{3}$ \\ Aimeric Courville, ${ }^{2}$ Rosa Di Felice, ${ }^{4,5}$ Zlatko Sitar, ${ }^{3}$ Philippe Vennéguès, ${ }^{2}$ and Martin Albrecht ${ }^{1}$ \\ ${ }^{1}$ Leibniz Institute for Crystal Growth, Max-Born-Strasse 2, 12489 Berlin, Germany \\ ${ }^{2}$ Centre de Recherche sur l'HétéroEpitaxie et ses Applications, \\ CNRS-CRHEA, rue Bernard Grégory, 06560 Valbonne, France \\ ${ }^{3}$ Material Science and Engineering, North Carolina State University, \\ 1001 Capability Drive, Raleigh, North Carolina 27695-7919, USA \\ ${ }^{4}$ Center S3, CNR Institute of Nanoscience, Via Campi 213/A, 41125 Modena, Italy \\ ${ }^{5}$ Department of Physics, University of Southern California, Los Angeles, California 90033, USA \\ (Received 14 December 2015; revised manuscript received 4 April 2016; published 3 May 2016)
}

\begin{abstract}
Controlling the polarity of polar semiconductors on nonpolar substrates offers a wealth of device concepts in the form of heteropolar junctions. A key to realize such structures is an appropriate buffer-layer design that, in the past, has been developed by empiricism. GaN or $\mathrm{ZnO}$ on sapphire are prominent examples for that. Understanding the basic processes that mediate polarity, however, is still an unsolved problem. In this work, we study the structure of buffer layers for group-III nitrides on sapphire by transmission electron microscopy as an example. We show that it is the conversion of the sapphire surface into a rhombohedral aluminum-oxynitride layer that converts the initial N-polar surface to Al polarity. With the various $\mathrm{Al}_{x} \mathrm{O}_{y} \mathrm{~N}_{z}$ phases of the pseudobinary $\mathrm{Al}_{2} \mathrm{O}_{3}-\mathrm{AlN}$ system and their tolerance against intrinsic defects, typical for oxides, a smooth transition between the octahedrally coordinated $\mathrm{Al}$ in the sapphire and the tetrahedrally coordinated $\mathrm{Al}$ in AlN becomes feasible. Based on these results, we discuss the consequences for achieving either polarity and shed light on widely applied concepts in the field of group-III nitrides like nitridation and low-temperature buffer layers.
\end{abstract}

DOI: 10.1103/PhysRevApplied.5.054004

\section{INTRODUCTION}

The most important compound semiconductors for applications in optoelectronics crystallize in the sphalerite or wurtzite structure, which contain polar axes. Polarity severely influences their physical properties with a strong impact on electronic or optoelectronic devices through the presence of pyroelectric and piezoelectric fields in strained epitaxial layers [1,2]. If domains with defined sizes and different polarities can be grown in parallel on a nonpolar substrate, fascinating perspectives for device concepts based on polarity engineering are opened. Phase-matching structures for nonlinear optical devices [3,4] or the realization of two-dimensional electron gases at heteropolar interfaces for electronic applications are examples [5,6]. Another field is compound semiconductor nanowires that grow stably along the polar axes. Nanowires show high crystalline perfection and are free of extended defects even on foreign substrates, but polarity control is still a challenging issue that essentially influences impurity concentrations and the incorporation of constituents of alloys [7].

Though the polarity is of crucial importance, existing concepts of polarity control are based on empiricism, and a basic understanding of the elementary mechanisms behind it is still missing. A common concept is the deposition of a

\footnotetext{
*stefan.mohn@ikz-berlin.de
}

buffer layer between the polar layer and the nonpolar substrate $[8,9,10,11]$. For the growth of group-III nitrides on sapphire, the development of an appropriate AlN buffer layer by Amano and Akasaki and later of GaN buffer layers by Nakamura is among the important breakthroughs that paved the way to device-grade materials [8,9]. In group-III nitrides, such buffer layers are formed in three steps: First, the surface of the sapphire is exposed to ammonia, commonly known as nitridation. Then a thin layer of AlN [8] or GaN [9] is deposited onto the nitridated surface at relatively low growth temperatures, which is subsequently annealed at high temperatures. The importance of the nitridation step for improving structural and optical properties has first been pointed out by Kawakami et al. [12] and later related to polarity control by Rouvière et al. [13]. X-ray photoelectron spectroscopy (XPS) shows that nitridation converts the sapphire $\left(\mathrm{Al}_{2} \mathrm{O}_{3}\right)$ surface stepwise to AIN (e.g., in Refs. [14-16]) by a continuous substitution of oxygen atoms in the sapphire substrate by nitrogen. A variety of aluminum-oxynitride $\left(\mathrm{Al}_{x} \mathrm{O}_{y} \mathrm{~N}_{z}\right)$ phases, in particular, those with spinel-type structure, containing both octahedrally and tetrahedrally coordinated $\mathrm{Al}$ [17], allow a smooth transition between sapphire with octahedrally coordinated $\mathrm{Al}$ and $\mathrm{AlN}$ with tetrahedrally coordinated $\mathrm{Al}[14,15,16,18]$. Accordingly, a coupled diffusion process of $\mathrm{H}, \mathrm{O}$, and $\mathrm{N}$ drives the exchange process, with the oxygen out-diffusion being the rate-limiting step $[15,19]$. 
While the chemical processes of the nitridation step are studied in detail, very little and contradictory work is presented on structural aspects that mediate polarity. This concerns the crystalline structure of the nitridation layer and the interface between the nitridation layer and the sapphire substrate. Structural models of the interface between the buffer layer and the sapphire substrate are presented in the form of rigid ball-and-stick models $[20,21,15]$. Di Felice and Northrup were among the first to use the density-functional theory (DFT) to get more insight into the problem [22]. They show that in the growth of AlN on sapphire Al-rich growth conditions lead to an $\mathrm{Al}$ polar layer, while $\mathrm{N}$ polarity is expected for $\mathrm{N}$-rich growth conditions.

In this work, we present a detailed study on the structure of different buffer layers with respect to the polarity control by aberration-corrected high-resolution transmission electron microscopy (HRTEM) as well as scanning transmission electron microscopy (STEM). We show that nitridation of the sapphire substrate results in a rhombohedral $\mathrm{Al}_{x} \mathrm{O}_{y} \mathrm{~N}_{z}$ layer that converts the initially N-polar nucleated $\mathrm{AlN}$ to $\mathrm{Al}$ polarity. The $\mathrm{Al}_{x} \mathrm{O}_{y} \mathrm{~N}_{z}$ layer, however, dissolves under high-temperature growth conditions typical for group-III nitrides, and the initially N-polar AlN is reestablished and acts as a N-polar template. It is the role of the low-temperature group-III-nitride buffer, itself stable at a high temperature in the growth environment for group-III nitrides, to preserve the metal polarity established by the unstable $\mathrm{Al}_{x} \mathrm{O}_{y} \mathrm{~N}_{z}$ layer.

\section{EXPERIMENTAL METHODS}

\section{A. Samples}

The investigated layers are metal-polar AlN and GaN templates and a N-polar AlN template grown by metalorganic chemical vapor deposition (MOCVD). Metal-polar layers are obtained by the deposition of a thin GaN or AlN buffer layer at low temperatures on nitrided sapphire surfaces, while no buffer layer is used for N-polar templates. Epi-ready $c$-plane sapphire substrates, which are first annealed in vacuum, then etched in hydrogen at $1050^{\circ} \mathrm{C}$, and subsequently nitrided in $\mathrm{NH}_{3}$, are used for all layers.

The AlN templates are grown at low pressure with the growth reactor operated at approximately 27 mbar. The nitridation of the substrate takes place in $\mathrm{NH}_{3}$ ambient with $\mathrm{N}_{2}$ as the carrier gas for $4 \mathrm{~min}$ at $950^{\circ} \mathrm{C}$ and $10 \mathrm{~min}$ at $1090^{\circ} \mathrm{C}$ for the metal-polar and the $\mathrm{N}$-polar template, respectively. The low-temperature AlN buffer layer, used only for the metal-polar template, is deposited at $650{ }^{\circ} \mathrm{C}$ with $\mathrm{NH}_{3}$ and trimethylaluminum as a precursor and a V:III ratio of 13000 . The buffer is then subsequently annealed in $\mathrm{NH}_{3}+\mathrm{N}_{2}$ ambient for $15 \mathrm{~min}$ at $1050{ }^{\circ} \mathrm{C}$. The thickness of the annealed buffer layer is around $20 \mathrm{~nm}$. In both cases, 150-200 $\mathrm{nm}$ of AlN are grown at temperatures around $1100{ }^{\circ} \mathrm{C}$ and a V:III ratio of 2000 to finalize the templates.

The $\mathrm{GaN}$ template is grown at atmospheric pressure with a total reactor pressure of $800 \mathrm{mbar}$. The sapphire surface is nitridated for 7 min at $1080^{\circ} \mathrm{C}$ with $\mathrm{H}_{2}$ as the carrier gas. The low-temperature $\mathrm{GaN}$ buffer-layer deposition takes place at $580^{\circ} \mathrm{C}$ with trimethylgallium as the metal precursor with a V:III ratio of 16000 . Annealing takes place in $\mathrm{NH}_{3}+\mathrm{H}_{2}$ ambient during the temperature rise to the final epitaxial temperature of $1080^{\circ} \mathrm{C}$. The thickness of the template is around $30 \mathrm{~nm}$.

For TEM investigations, cross-sectional samples of these layers are prepared along $\langle 11-20\rangle$ and $\langle 1-100\rangle$ lattice directions. The samples are prepared by tripod or wedge polishing with diamond lapping foils and finally thinned to electron transparency by Ar ion milling with a Gatan Precision Ion Polishing System (PIPS) using acceleration voltages between 4.0 and $0.2 \mathrm{kV}$.

\section{B. Transmission electron microscopy}

For our investigations, a FEI Titan 80-300 TEM, operated at $300 \mathrm{kV}$ equipped with corrector for spherical aberrations $\left(C_{s}\right)$ of the objective lens, is utilized. For optimal contrast during the HRTEM measurements, imaging conditions with a small negative $C_{s}$ value and positive defocus are chosen, i.e., $C_{s}=-8 \mu \mathrm{m}$ and $+5 \mathrm{~nm}$ defocus. With these imaging conditions and sufficient thin samples (usually below $8 \mathrm{~nm}$ ), atomic resolution in the $\langle 11-20\rangle$ projection of the AlN layer is achieved even for light elements, such as $\mathrm{N}$ and $\mathrm{O}$, and atoms appear bright [23]. We are able to distinguish between $\mathrm{N}$ and $\mathrm{Al}$ atomic columns, the latter appearing at a higher intensity. This allows a distinct analysis of the film polarity. A showcase for this purpose can be found in Fig. 1, where the measured $\mathrm{N}$-polar AlN and a corresponding phase-contrast simulation are shown, respectively. Besides HRTEM measurements, results obtained by STEM high-angle annular dark-field (HAADF) are presented, where the contrast is

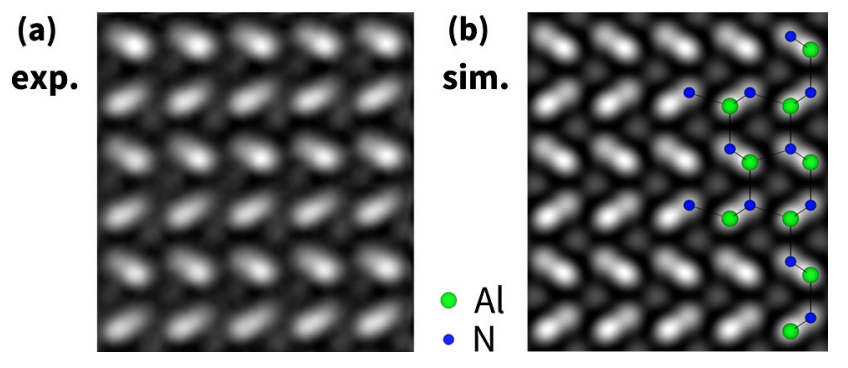

FIG. 1. Comparison between experimental and simulated HRTEM images of N-polar AIN. (a) Experimental image obtained by using a slightly negative spherical aberration $\left(C_{s}\right)$ and a small positive defocus and (b) simulated contrast by the multislice approach for $C_{s}=-8 \mu \mathrm{m},+5 \mathrm{~nm}$ defocus, and a sample thickness of $4.97 \mathrm{~nm}$. Al appears at a higher intensity, $\mathrm{N}$ at a lower intensity. 
mainly given by the atomic number ( $Z$ contrast) of the columns. Lower intensity represents a lower atomic number or, alternatively, can be a hint to a reduced number of atoms due to vacancies in the atomic column. For STEM-HAADF imaging, we use a semiconvergence angle of the incident electron probe of $9 \mathrm{mrad}$, resulting in a spatial resolution of about $0.13 \mathrm{~nm}$, and a semiacceptance angle of the annular dark-field detector (model 3000, Fishione) of $35 \mathrm{mrad}$.

The HRTEM images presented in this work are composed of several (commonly around 20) HRTEM images, which are recorded continuously with identical imaging conditions. After a correction of drift, which might occur during the image acquisition, the intensities of the single images are summed up and the intensity of the resulting image is normalized. Images obtained by STEM-HAADF are usually adjusted to have maximum contrast. In some cases, the STEM-HAADF patterns are compositions of periodically occurring patches with similar features from a single image, which are superimposed.

Images recorded by TEM cannot be interpreted directly but have to be compared with simulated contrast patterns based on known structural models. HRTEM patterns are simulated with the multislice [24] approach using the EMS software package [25]. The simulation parameters corresponding to the experimental conditions are $300 \mathrm{kV}$ for the electron beam acceleration voltage, a semiconvergence angle $\alpha=0.3 \mathrm{mrad}$, a defocus spread $\Delta f=2.9 \mathrm{~nm}$, a spherical aberration $C_{s}=-8 \mu \mathrm{m}$, and a slight overfocus of $5 \mathrm{~nm}$. The contrast patterns shown in this work are for sample thicknesses between 4.5 and $5 \mathrm{~nm}$, which gives us the best match between simulation and experiment. STEM image simulations are performed by using a parallelized multislice approach [26] with the frozen-phonon approximation and a total number of 30 different frozen-phonon configurations. The structure used for the STEM simulations in Fig. 5(b) extends over 18 monolayers along the $c$ direction [four monolayers of the $\mathrm{Al}_{x} \mathrm{O}_{y} \mathrm{~N}_{z}$ IDB and seven monolayers of AlN on each side] and $24 a$ lattice parameters (approximately $7.5 \mathrm{~nm}$ ) in the direction of the electron beam. Cation sites in the four inversion domain boundary (IDB) layers are statistically occupied within the depth of the supercell with either $\mathrm{Al}$ atoms or vacancies according to the average occupancy of the columns. Imaging parameters of the simulation (semiconvergence angle, detector acceptance angle, etc.) are used according to experimental conditions.

\section{DFT calculations}

For the identification of the atomic configuration at the interface between the sapphire and AlN, we use structural models obtained by periodic-supercell planewave DFT calculations with the QUANTUM-ESPRESSO software [27], using a 25-Ry kinetic energy cutoff and the Perdew, Burke, and Ernzerhof (PBE) exchange-correlation functional [28]. The electron-ion interaction is described with non-norm-conserving pseudopotentials [29]. With this computational approach and converged Monkhorst-Pack grids for Brillouin zone (BZ) summations, the lattice constants of bulk $\mathrm{Al}_{2} \mathrm{O}_{3}$ (wurtzite AlN) are $a=$ $0.479 \mathrm{~nm}$ and $c / a=2.76(a=0.312 \mathrm{~nm}, c / a=1.61$, and $u=0.3808$ ), which agree with the experimental data (slight overestimate of $0.5 \%$ for $a$ and $1 \%-2 \%$ for $c$, typical of PBE). BZ summations in interface calculations are approximated with six inequivalent $\mathbf{k}$ points $(4 \times 4 \times 1$ Monkhorst-Pack grid). The lattice constant of the $1 \times 1$ sapphire lattice (approximately 13\% interface strain) is imposed, and symmetric supercells along the $c$ axis are used, with a vacuum thickness of about $2 \mathrm{~nm}$ perpendicular to the $c$ plane, to avoid spurious interactions between neighboring replicas. The substrate consists of a bulk $\mathrm{Al}_{2} \mathrm{O}_{3}$ unit (six $\mathrm{O}$ layers, separated by $\mathrm{Al}$ double layers with $2 / 3$ occupation [Figs. 4(a) and 4(c)]): We check that doubling the sapphire thickness along the $c$ axis does not change the interface structure and energetics. The films contains 1-2 AlN layers, but only those with two film layers are used for multislice simulations. The surface termination is one $\mathrm{Al}$ plane, for optimizing surface energetics: The surface termination, visible in Figs. 4(a) and 4(c), does not interfere with the analysis of film polarity. [22]. The structures are fully relaxed by vanishing the Hellmann-Feynman DFT forces within a precision of $0.2 \mathrm{eV} / \mathrm{nm}$. Note that at such strained interfaces it costs $1.1(1.3) \mathrm{eV} /$ pair to add the second AlN layer in the film; the overall energetics between $\mathrm{N}$ - and Al-polar films is consistent with the earlier analysis with thinner films and less-precise DFT details.

\section{RESULTS AND DISCUSSION}

Figures 2(a) and 3 show cross-sectional images of the interface between the epitaxial AlN film and the sapphire substrate in the two major projection directions, i.e., $\mathrm{AlN}_{\langle 1-100\rangle} \| \mathrm{Al}_{2} \mathrm{O}_{3\langle 11-20\rangle}$ [Fig. 2(a)] and $\mathrm{AlN}_{\langle 11-20\rangle} \|$ $\mathrm{Al}_{2} \mathrm{O}_{3\langle 1-100\rangle}$ (Fig. 3). Within the range shown in Fig. 3, the film consists of the nitridation layer and the annealed low-temperature AlN buffer layer. The in-plane rotation of the layer by $30^{\circ}$ with respect to the sapphire substrate is due to the lower lattice mismatch in this orientation relationship and is widely described in the literature (see, e.g., Ref. [30] and references therein). The interface is atomically abrupt, as reported by other authors $[31,21,15,32]$.

\section{A. Interface structure}

Before analyzing the polarity of the layer in more detail, we focus on the interface structure. We differentiate between "Al-polar" [Fig. 4(a)] and "N-polar" [Fig. 4(c)] interface models as introduced by Di Felice and Northrup [22]. The atomic arrangement at the interface defines the polarity of the nucleating AlN. The DFT calculations are performed for AlN layers coherently strained to sapphire, 


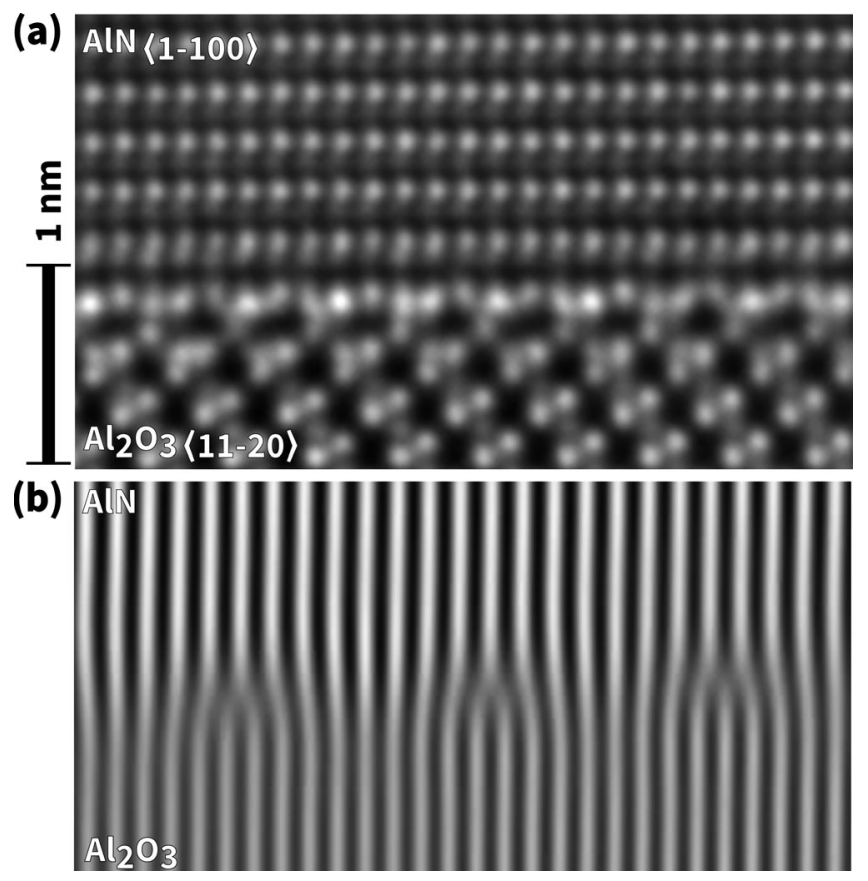

FIG. 2. (a) HRTEM image of the interface structure of AIN on sapphire $\left(\mathrm{Al}_{2} \mathrm{O}_{3}\right)$ along $\mathrm{AlN}\langle 1-100\rangle$. The interface is atomically abrupt and shows a regular arrangement of misfit dislocations. (b) Bragg-filtered image of (a) showing the misfit dislocation network.

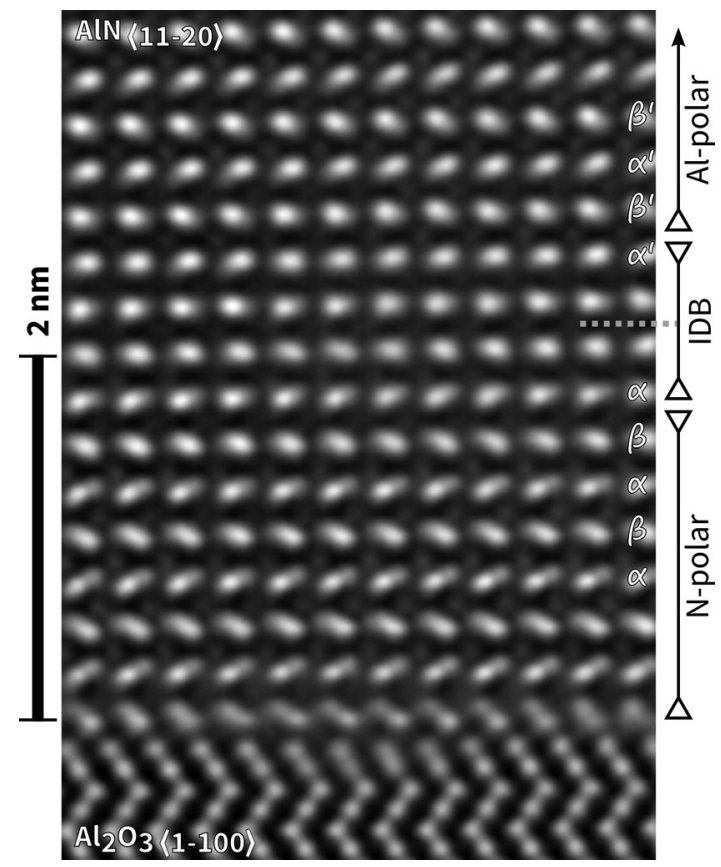

FIG. 3. HRTEM image of the AIN/sapphire interface along $\mathrm{AlN}_{\langle 11-20\rangle}$. The polarity of the film changes approximately $2 \mathrm{~nm}$ above the interface from $\mathrm{N}$ - to Al-polar (as labeled). The stacking of the lattice is marked by $\alpha \beta$ for the N-polar and $\alpha^{\prime} \beta^{\prime}$ for the Alpolar material. The layer, which mediates the change of polarity, violates the stacking sequence. while our group-III-nitride layers are completely relaxed by misfit dislocations at the interface as shown in Fig. 2(b). We therefore compare experimental and simulated images at sites between two misfit dislocations, where both the substrate and the layer are in coincidence. At these coincidence sites, the in-plane atomic displacements that occur due to the strain field of adjacent misfit dislocations, annihilate. A comparison of the experimental interface [Fig. 4(b)] and simulated interface models [Figs. 4(a) and $4(\mathrm{c})$ ] reveals two important experimental findings: (i) In the $\mathrm{AlN}_{\langle 11-20\rangle}$ projection [Fig. 4(b), (bottom row)], the first AlN layers appear N-polar; (ii) in the $\mathrm{AlN}_{\langle 1-100\rangle}$ projection [Fig. 4(b), (top row)], we find a characteristic line-shaped pattern [marked in Fig. 4(b)], which matches the phase contrast of a linear arrangement of O-Al-N atoms in the N-polar interface simulation [marked likewise in Fig. 4(c)]. In the Al-polar interface, atoms of the first two monolayers of AlN are displaced. In the $\mathrm{AlN}_{\langle 1-100\rangle}$ projection, an aluminum position is strongly buckled towards the interface and appears together with the underlying nitrogen in the
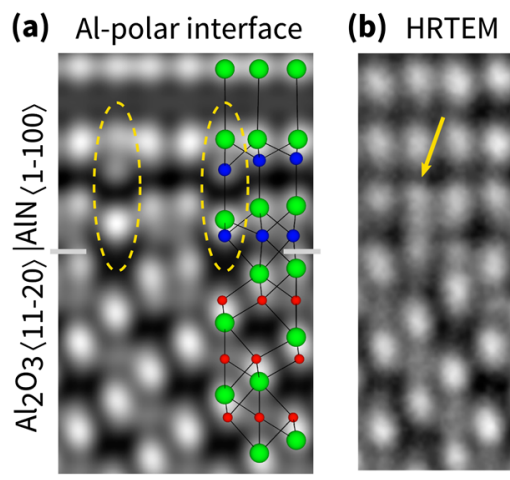

(c) N-polar interface
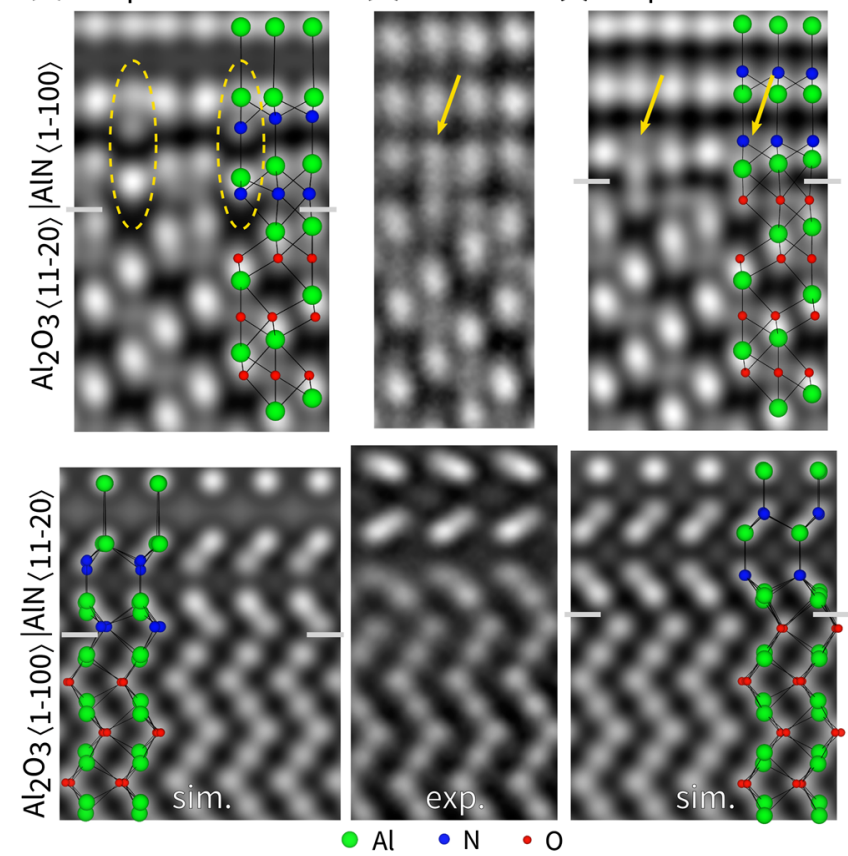

FIG. 4. Analysis of the interface structure of AIN on sapphire. Theoretically calculated atomic configurations of the Al-polar [column (a)] and the N-polar [column (c)] interface are represented by ball-and-stick models with corresponding contrast simulations along the two major projection directions (top row, $\mathrm{AlN}_{\langle 1-100\rangle}$; bottom row, $\mathrm{AlN}_{\langle 11-20\rangle}$ ) The interface is indicated by the small gray lines. Column (b) shows the interface measured by HRTEM. In the $\mathrm{AlN}_{\langle 1-100\rangle}$ projection (top row), characteristic arrangements are marked: The yellow arrow in (b) and (c) points to the linear stacking of O-Al-N atoms. In column (a) adjacent $\mathrm{Al}$ and $\mathrm{N}$ columns are visible as a characteristic bright blob, and in the second monolayer distinct $\mathrm{Al}$ and $\mathrm{N}$ positions are encircled. 
phase-contrast image as a bright blob. Above this arrangement, in the second layer, the $\mathrm{N}$ is shifted and appears with the corresponding $\mathrm{Al}$ as a dumbell in the phase-contrast image. Both features, encircled in Fig. 4(a)), are not seen in the experiment. Thus, our analysis clearly reveals a N-polar interface, as expected from $a b$ initio studies, where nitridation is described as the N-rich limit of growth, with sapphire as the source of $\mathrm{Al}$ [22].

\section{B. Inversion domain boundary}

Let us now follow the polarity of the film upon further growth as shown in Fig. 3: The first eight AlN layers appear to be N-polar, while the two following monolayers do not permit any assignment of polarity. After these intermediate layers, the polarity changes suddenly to $\mathrm{Al}$ polarity. This abrupt transition of the polarity comes along with a discontinuity in the stacking sequence: In the N-polar layer, the stacking sequence follows an $\alpha \beta$ (i.e., $A a B b \ldots)$ stacking as expected for the wurtzite lattice and ends with an $\alpha$-stacked layer. The first Al-polar layer starts also with an $\alpha^{\prime}$-stacked layer. In consequence, the two layers, that mediate the polarity change, introduce an error in the stacking sequence. A conventional stacking fault, i.e., an inclusion of layers with cubic stacking, would cause an in-plane translation, which is not observed here and can (a) HRTEM

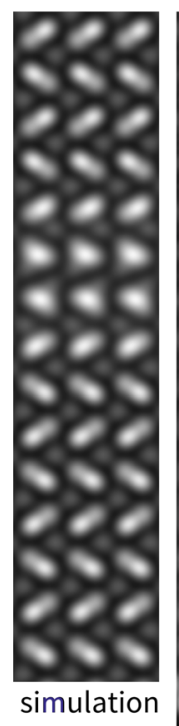

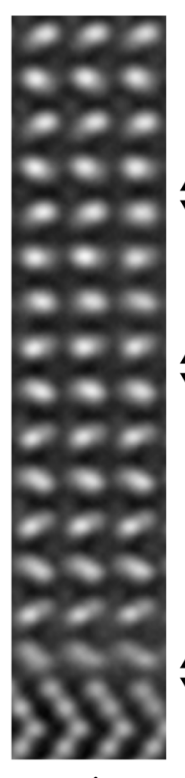

experiment

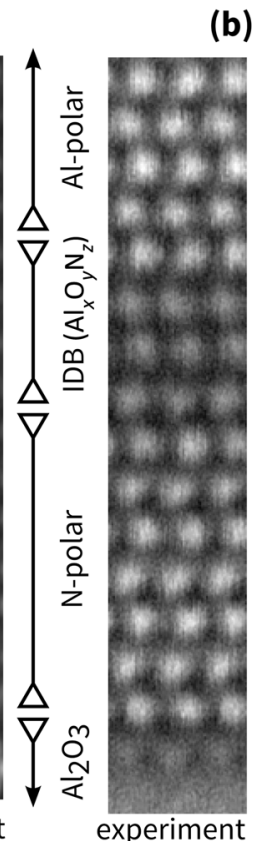

(b) STEM

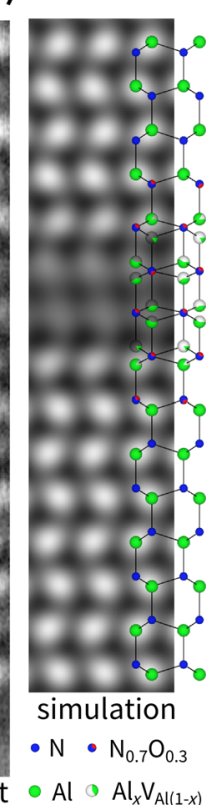

FIG. 5. Comparison of HRTEM (a) and STEM (b) images of the AlN layer above the sapphire interface including the planar inversion domain boundary. Experimental images are confronted to image simulations, which are based on a model of an $\mathrm{Al}_{x} \mathrm{O}_{y} \mathrm{~N}_{z}$ IDB in an AIN matrix. A ball-and-stick representation of this model is shown next to the STEM simulation. A detailed description of the model is presented in Fig. 6. be therefore excluded. Since the film polarity is switched around these layers, we denote them as the planar IDB.

To gain additional information on these structures, we present STEM-HAADF images [see Fig. 5(b)] of identical samples. We cannot directly resolve $\mathrm{Al}$ and $\mathrm{N}$ atomic columns by STEM-HAADF $Z$ contrast. Therefore, we analyze the polarity from the tunnel position as proposed by Rouvière et al. [33]. The most important findings from these investigations are (i) a change of the lattice polarity caused by a planar IDB confirming independently our HRTEM investigations and (ii) a reduced intensity at the planar IDB.

\section{C. $\mathrm{Al}_{x} \mathrm{O}_{y} \mathbf{N}_{z}$ model}

In the following, we develop a model for the planar IDB based on these experimental findings. Since evidence for the formation of $\mathrm{Al}_{x} \mathrm{O}_{y} \mathrm{~N}_{z}$ during the early stages of the nitridation process has been found by XPS [14,15,16,19], we consider the various $\mathrm{Al}_{x} \mathrm{O}_{y} \mathrm{~N}_{z}$ phases of the pseudobinary $\mathrm{Al}_{2} \mathrm{O}_{3}$-AlN system as an obvious choice. Phases range from the rhombohedral corundum structure and defective spinel-type structures in the $\mathrm{Al}_{2} \mathrm{O}_{3}$-rich domain to rhombohedral and hexagonal phases in the AlN-rich part of the system. The phase diagram and detailed information about the phases can be found in the work of McCauley et al. [18] and references therein. The most natural choice for our purpose is the rhombohedral R21 and R27 phases in the AlN-rich part. These phases have been recently synthesized in crystalline form and analyzed with the Rietveld method by Asaka et al. [34,35]. The 27R (or $\mathrm{Al}_{9} \mathrm{O}_{3} \mathrm{~N}_{7}$ ) phase, for instance, has an enormous $c$-lattice parameter of $7.2 \mathrm{~nm}$ and a layered superstructure based on AlN with different polarity and planar IDBs. We may focus our attention on a subsection of the lattice that changes the polarity from "N/O" to "Al." It ranges over four monolayers and essentially consists of two interpenetrating "N/O-polar" and "Al-polar" wurtzite-type lattices that share a common anion sublattice. Along $\langle 0001\rangle$, the N/O-polar cations fade out with stepwise reduced occupancy, while accordingly the occupancy of the Al-polar cations likewise increases (for a detailed model, see Refs. [34,35]). Based on a model with this IDB inside a matrix of AlN (Fig. 6), we perform contrast simulations for phase-contrast imaging as well as for Z-contrast STEM imaging. We confront the results of these simulations with corresponding experimental images in Fig. 5. In the HRTEM experimental images [Fig. 5(a)], the first and the last layer of the IDB are hard to distinguish from pure AlN dumbbells. This similarity is due to the fact that the N-polar cations at the lower interface and the Alpolar cations at the upper interface have a dominating occupation (approximately 83\%). In the two central layers, both cationic positions have nearly equal occupancy (like approximately $60 \%$ to approximately $40 \%$ ), and thus the polarity cannot be determined in the images. Comparing the experimental and simulation data in Fig. 5(a), we find 


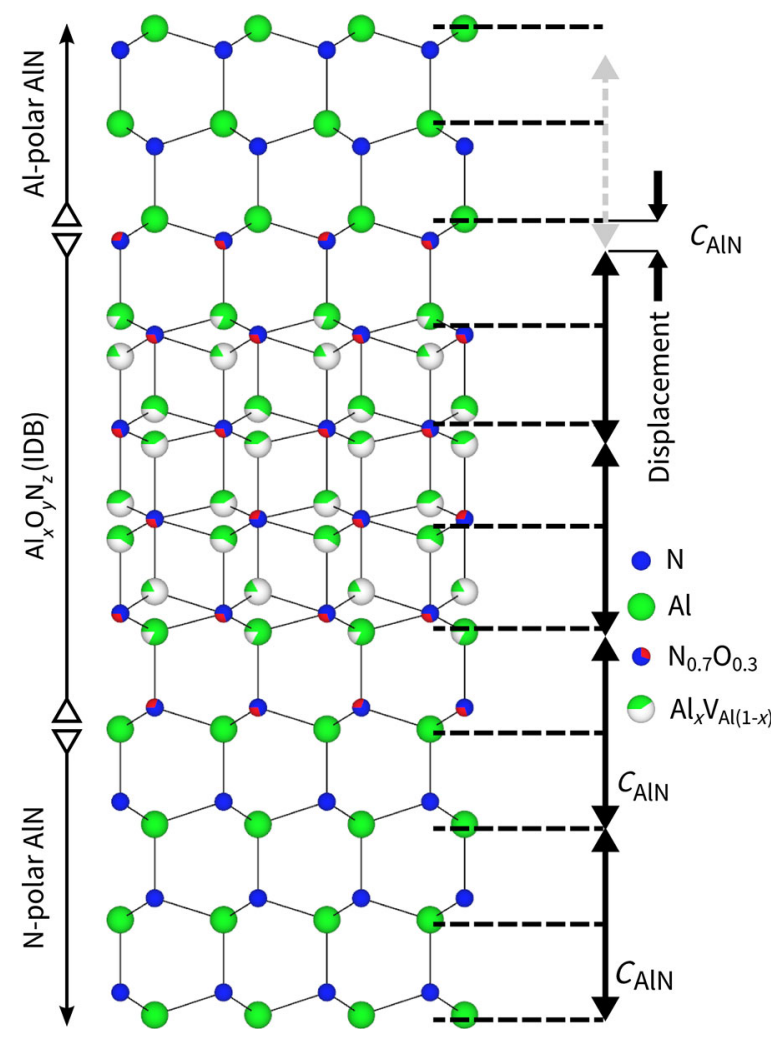

FIG. 6. Ball-and-stick model of the $27 \mathrm{R}-\mathrm{Al}_{9} \mathrm{O}_{3} \mathrm{~N}_{7}$ IDB, responsible for the switch of N/O- to Al-polar lattice, embedded in an AlN matrix. The anions within the IDB consist of $70 \% \mathrm{~N}$ and $30 \%$ O. The cation positions in the IDB, displayed as big circles with different filling ratios, are occupied by $\mathrm{Al}$ and $\mathrm{Al}$ vacancies $\left(V_{\mathrm{Al}}\right)$, where the $\mathrm{Al}$ content of the column equals the filled fraction of the circle. The IDB affects the distances of the lattice planes along $\langle 0001\rangle$. The weighted positions of the cations, marked by the dashed lines, are evaluated as out-of-plane displacement $\Delta c_{\mathrm{AlN}}$ as schematically shown.

nearly perfect agreement. Turning to the STEM-HAADF images shown in Fig. 5(b), the reduced intensity of the IDB, caused by the presence of vacancies, agrees even on a quantitative scale with that of the experimental images.

Finally, we evaluate the displacement of atomic planes with respect to a perfect AlN lattice (see the schematic illustration in Fig. 6). We make use of the fact that, in the proposed structure, the cationic positions of the Al-polar lattice are shifted against the N/O-polar lattice by approximately $c_{\mathrm{AIN}} / 4$ along $\langle 0001\rangle$. We evaluate the shift obtained from the intensity maxima [36] in the experimental STEM-HAADF images and compare them to the shifts measured in the respective simulation. Since Al, O, and $\mathrm{N}$ positions cannot be resolved, the STEM-HAADF contrast pattern reveals a weighted-average position of these atomic columns, including the $\mathrm{Al}$ vacancies. Figure 7 shows the theoretical displacement of the cationic positions with respect to the wurtzite-type reference lattice of the $\mathrm{N}$ polar part of the layer and the displacement of the contrast maxima in the simulated and the experimental STEM

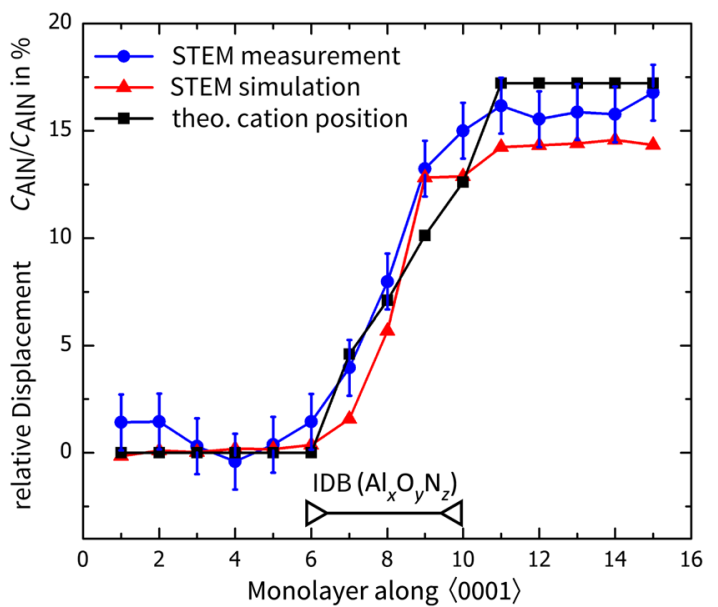

FIG. 7. Comparison of the out-of-plane displacement $\left(\Delta c_{\mathrm{AIN}}\right.$ along $\langle 0001\rangle$ ) of the weighted cation positions of the $\mathrm{Al}_{x} \mathrm{O}_{y} \mathrm{~N}_{z}$ IDB with respect to the surrounding AIN lattice and of intensity maxima in experimental and simulated STEM images of the lowtemperature AlN buffer layer. Simulations are based on the model shown in Fig. 6.

images. Again, the values obtained from simulated and experimental images agree well with and correspond to the theoretical displacement, which predicts a shift of approximately $17 \%$ between the two polar lattices.

\section{Role of the low-temperature buffer layer}

To prove that the Al polarity is established during the nitridation and to shed light on the role of the lowtemperature buffer, whose importance is pointed out by Amano et al. [8] and Nakamura [9], we study two additional samples by HRTEM and STEM. In one sample, a $\mathrm{GaN}$ buffer is deposited at $580^{\circ} \mathrm{C}$ on top of nitridated sapphire [Fig. 8(a)], and, in the other sample, an AlN epilayer is grown at $1100^{\circ} \mathrm{C}$ on nitridated sapphire without any additional buffer [Fig. 8(b)]. In the case of the lowtemperature GaN buffer, we can easily distinguish between the layer that forms during nitridation and the GaN buffer by Z-contrast imaging. Combining information from STEM and HRTEM images reveals that the nitridation layer, embedded between the sapphire and the lowtemperature $\mathrm{GaN}$ buffer, consists of wurtzite N-polar AlN, located right above the sapphire interface, and $\mathrm{Al}_{x} \mathrm{O}_{y} \mathrm{~N}_{z}$, layered between the N-polar AlN and the GaN buffer. The $\mathrm{Al}_{x} \mathrm{O}_{y} \mathrm{~N}_{z}$ appears in Z-contrast STEM by reduced intensity compared to the underlying AIN and shows in HRTEM the characteristic triangular-shaped intermediate monolayers around a planar IDB. Both layers, the N-polar AlN and the $\mathrm{Al}_{x} \mathrm{O}_{y} \mathrm{~N}_{z}$, which trace back to the nitridation process, are very similar to those shown above in the sample with the low-temperature AlN buffer. As seen in the HRTEM images, the GaN buffer grows metal-polar from the beginning and shows a high density of stacking faults [e.g., in Fig. 8(a), the uppermost two monolayers] 
(a) low-temp. GaN buffer

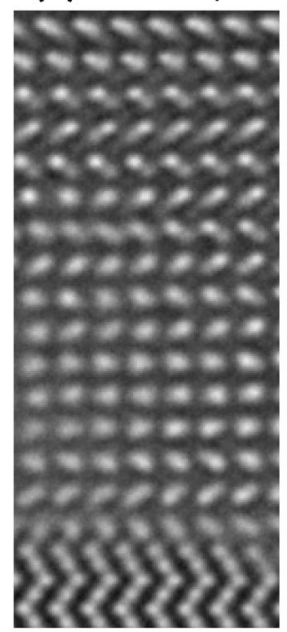

HRTEM

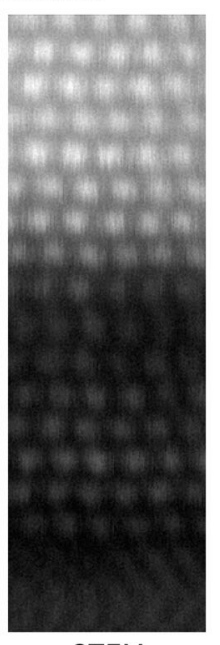

STEM (b) high-temp. AlN layer

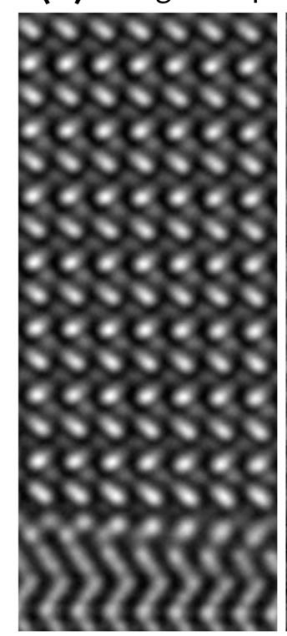

HRTEM

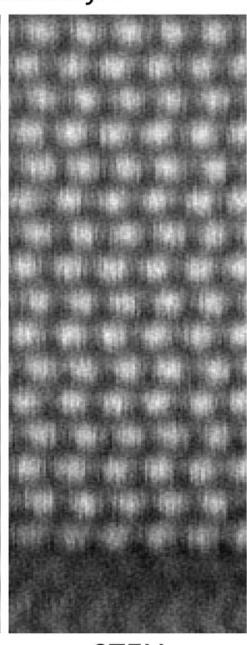

STEM (c)

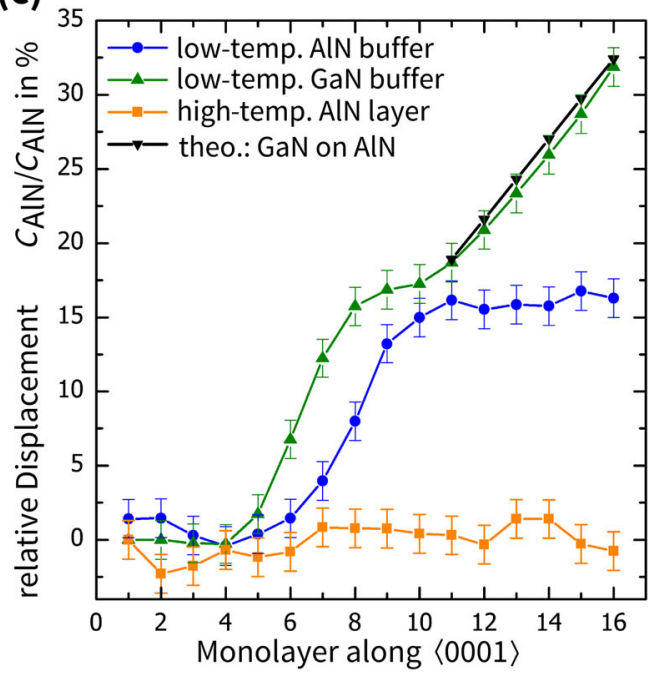

FIG. 8. HRTEM and STEM images of (a) a low-temperature GaN buffer layer and (b) an AlN layer grown at high temperature onto nitrided sapphire. In (a), the GaN layer shows up at high intensity in the STEM image, while the IDB is revealed by lower intensity. In (b), the layer starts to grow N-polar at the interface between AlN and sapphire. (c) Comparison of the displacements of the intensity maxima with respect to AlN obtained from the evaluation of the STEM images for low-temperature GaN and AlN buffer layers and a high-temperature AlN layer grown on nitrided sapphire. The displacement characteristic for the $\mathrm{Al}_{x} \mathrm{O}_{y} \mathrm{~N}_{z}$ layer is revealed in layers grown at low temperatures but is not observed in the layer grown at high temperature. The displacement for the low-temperature GaN layer increases linear due to the higher lattice constant of GaN compared to AlN.

due to the limited surface diffusion at low-temperature growth. An analysis of the lattice displacement, shown in Fig. 8(c), confirms these observations. After four monolayers of AlN with no displacement, a strong shift of the atomic planes reveals an $\mathrm{Al}_{x} \mathrm{O}_{y} \mathrm{~N}_{z}$ interlayer with the characteristic displacement of approximately 17\%. It is followed by a linear increase of the displacement, which matches the larger lattice constant of $\mathrm{GaN}$ as indicated by the theoretical curve for $\mathrm{GaN}$ on AlN. These observations clearly indicate that $\mathrm{N}$-polar $\mathrm{AlN}$ and $\mathrm{Al}_{x} \mathrm{O}_{y} \mathrm{~N}_{z}$, which converts the polarity, form during the nitridation of the sapphire surface. Metal-polarity is therefore mediated during nitridation, regardless of whether a low-temperature GaN or AlN buffer is deposited.

Let us now turn to the other sample, where a hightemperature AlN epilayer is grown directly on nitrided sapphire [Fig. 8(b)]. With this growth process, we obtain a $\mathrm{N}$-polar AlN template. We see neither in the STEMHAADF images a layer with reduced intensity characteristic for the planar IDB nor the change of polarity in HRTEM or STEM-HAADF images. Accordingly, this layer shows no notable displacement as displayed in Fig. 8(c). Since the nitridation step of this sample is similar to that with a low-temperature buffer, we conclude that the $\mathrm{Al}_{x} \mathrm{O}_{y} \mathrm{~N}_{z}$ layer formed during nitridation has disappeared in the growth ambient typical for epitaxial growth of AlN, i.e., the elevated temperatures and the increased use of a metal precursor. From the facts that metal polarity is established through $\mathrm{Al}_{x} \mathrm{O}_{y} \mathrm{~N}_{z}$ formation during nitridation and that no $\mathrm{Al}_{x} \mathrm{O}_{y} \mathrm{~N}_{z}$ layer is observed after high-temperature growth of the N-polar film, we conclude that it is the role of the lowtemperature buffer layer to preserve the metal-polarity, established by the $\mathrm{Al}_{x} \mathrm{O}_{y} \mathrm{~N}_{z}$ layer, and protect it against dissolution in high-temperature epitaxial environments. This means that the growth temperature of the first epitaxial deposited group-III nitride is a crucial parameter to establish either $\mathrm{N}$ or metal-polarity in group-III nitrides.

The annealing procedure of the low-temperature buffer is found to be another crucial parameter, which affects the structural perfection of the layer [37]. Our observations suggest that annealing of the flat low-temperature buffer leads to a three-dimensional surface that causes dislocation bending and thereby reduces the dislocation density. Excessive annealing of the low-temperature buffer, on the other hand, may lead to incomplete coverage of the $\mathrm{Al}_{x} \mathrm{O}_{y} \mathrm{~N}_{z}$ and therefore to the formation of mixed polarity. Taking the crucial role of $\mathrm{Al}_{x} \mathrm{O}_{y} \mathrm{~N}_{z}$ in controlling polarity into account, it is highly interesting to consider work that has been performed in the early period of group-III-nitride research. Kawakami, for instance, emphasizes the importance of residual oxygen in the nitridation layer for achieving high-quality material [12].

\section{CONCLUSIONS}

Our findings suggest that oxide substrates and oxidecontaining layers are very helpful in controlling the polarity on nonpolar substrates. The reason for this lies in the fact that oxides exhibit various phases with structures, which are tolerant against a high concentration of intrinsic atomic 
defects and in many cases contain tetrahedrally and octahedrally coordinated atoms in the same structure. An example from the case of oxides is the growth of $\mathrm{ZnO}$ on sapphire with a $\mathrm{MgO}$ buffer layer [10]. Chen et al. suggest that a phase change of the $\mathrm{MgO}$ buffer layer from a wurtzitelike phase at the sapphire interface to the equilibrium rocksalt structure would lead to trigger metal-polarity in the growth of $\mathrm{ZnO}$ on the nonpolar sapphire substrate. However, there is no direct evidence by TEM that these two layers are present, but $\mathrm{MgAl}_{2} \mathrm{O}_{4}$ is a prototype of the spinel structure [38], and we speculate that it possibly may form as an intermediate layer in this case.

\section{ACKNOWLEDGMENTS}

We thank Tobias Schulz, Thilo Remmele, and Detlef Klimm of the Leibniz-Institute for Crystal Growth for helpful discussions. We highly acknowledge discussions with J.E. Northrup. We acknowledge financial support from NSF (DMR-1108071) and DFG in the framework of the project Science of polar homo- and heterointerfaces of the Materials World Network and from GANEX (ANR-11LABX-0014). GANEX belongs to the public funded "Investissements d'Avenir" program managed by the French ANR agency.

[1] T. Takeuchi, C. Wetzel, S. Yamaguchi, H. Sakai, H. Amano, I. Akasaki, Y. Kaneko, S. Nakagawa, Y. Yamaoka, and N. Yamada, Determination of piezoelectric fields in strained GaInN quantum wells using the quantum-confined Stark effect, Appl. Phys. Lett. 73, 1691 (1998).

[2] M. Leroux, N. Grandjean, M. Laügt, J. Massies, B. Gil, P. Lefebvre, and P. Bigenwald, Quantum confined Stark effect due to built-in internal polarization fields in $(\mathrm{Al}, \mathrm{Ga}) \mathrm{N} / \mathrm{GaN}$ quantum wells, Phys. Rev. B 58, R13371 (1998).

[3] A. Chowdhury, H. M. Ng, M. Bhardwaj, and N. G. Weimann, Second-harmonic generation in periodically poled GaN, Appl. Phys. Lett. 83, 1077 (2003).

[4] J. Park, Y. Yamazaki, M. Iwanaga, S. Ahn, H. Jeon, T. Fujiwara, and T. Yao, Second harmonic generation in periodically polarity-inverted zinc oxide, Opt. Express 18, 7851 (2010).

[5] M. Stutzmann, O. Ambacher, M. Eickhoff, U. Karrer, A. Lima Pimenta, R. Neuberger, J. Schalwig, R. Dimitrov, P. Schuck, and R. Grober, Playing with polarity, Phys. Status Solidi B 228, 505 (2001).

[6] A. Aleksov, R. Collazo, S. Mita, R. Schlesser, and Z. Sitar, Current-voltage characteristics of $\mathrm{n} / \mathrm{n}$ lateral polarity junctions in GaN, Appl. Phys. Lett. 89, 052117 (2006).

[7] K. Hestroffer, C. Leclere, C. Bougerol, H. Renevier, and B. Daudin, Polarity of GaN nanowires grown by plasmaassisted molecular beam epitaxy on Si(111), Phys. Rev. B 84, 245302 (2011).

[8] H. Amano, N. Sawaki, I. Akasaki, and Y. Toyoda, Metalorganic vapor phase epitaxial growth of a high quality GaN film using an AlN buffer layer, Appl. Phys. Lett. 48, 353 (1986).
[9] S. Nakamura, GaN growth using GaN buffer layer, Jpn. J. Appl. Phys. 30, L1705 (1991).

[10] Y. Chen, S.-K. Hong, H.-J. Ko, V. Kirshner, H. Wenisch, T. Yao, K. Inaba, and Y. Segawa, Effects of an extremely thin buffer on heteroepitaxy with large lattice mismatch, Appl. Phys. Lett. 78, 3352 (2001).

[11] Y. Chen, H.-J. Ko, S.-K. Hong, and T. Yao, Layer-by-layer growth of $\mathrm{ZnO}$ epilayer on $\mathrm{Al}_{2} \mathrm{O}_{3}(0001)$ by using a $\mathrm{MgO}$ buffer layer, Appl. Phys. Lett. 76, 559 (2000).

[12] H. Kawakami, K. Sakurai, K. Tsubouchi, and N. Mikoshiba, Epitaxial growth of AlN film with an initial-nitriding layer on $\alpha-\mathrm{Al}_{2} \mathrm{O}_{3}$ substrate, Jpn. J. Appl. Phys. 27, L161 (1988).

[13] J. L. Rouvière, M. Arlery, R. Niebuhr, K. H. Bachem, and O. Briot, Transmission electron microscopy characterization of GaN layers grown by MOCVD on sapphire, Mater. Sci. Eng. B 43, 161 (1997).

[14] K. Uchida, A. Watanabe, F. Yano, M. Kouguchi, T. Tanaka, and S. Minagawa, Nitridation process of sapphire substrate surface and its effect on the growth of GaN, J. Appl. Phys. 79, 3487 (1996).

[15] Y. Cho, Y. Kim, E. R. Weber, S. Ruvimov, and Z. LilientalWeber, Chemical and structural transformation of sapphire $\left(\mathrm{Al}_{2} \mathrm{O}_{3}\right)$ surface by plasma source nitridation, J. Appl. Phys. 85, 7909 (1999).

[16] M. Losurdo, P. Capezzuto, G. Bruno, G. Namkoong, W. A. Doolittle, and A.S. Brown, Role of sapphire nitridation temperature on GaN growth by plasma assisted molecular beam epitaxy: Part II. Interplay between chemistry and structure of layers, J. Appl. Phys. 91, 2508 (2002).

[17] C. M. Fang, R. Metselaar, H. T. Hintzen, and G. de With, Structure models for $\gamma$-aluminum oxynitride from $a b$ initio calculations, J. Am. Ceram. Soc. 84, 2633 (2001).

[18] J. W. McCauley, P. Patel, M. Chen, G. Gilde, E. Strassburger, B. Paliwal, K. T. Ramesh, and D. P. Dandekar, AlON: A brief history of its emergence and evolution, J. Eur. Ceram. Soc. 29, 223 (2009).

[19] F. Dwikusuma and T.F. Kuech, X-ray photoelectron spectroscopic study on sapphire nitridation for GaN growth by hydride vapor phase epitaxy: Nitridation mechanism, J. Appl. Phys. 94, 5656 (2003).

[20] F. A. Ponce, J. S. Major, W. E. Plano, and D. F. Welch, Crystalline structure of AlGaN epitaxy on sapphire using AlN buffer layers, Appl. Phys. Lett. 65, 2302 (1994).

[21] N. Grandjean, J. Massies, Y. Martinez, P. Vennéguès, M. Leroux, and M. Laügt, GaN epitaxial growth on sapphire ( $\left.\begin{array}{llll}0 & 0 & 0 & 1\end{array}\right)$ : The role of the substrate nitridation, J. Cryst. Growth 178, 220 (1997).

[22] R. Di Felice and J. E. Northrup, Energetics of AlN thin films on the $\mathrm{Al}_{2} \mathrm{O}_{3}(0001)$ surface, Appl. Phys. Lett. 73, 936 (1998).

[23] C. L. Jia and K. Urban, Atomic-resolution measurement of oxygen concentration in oxide materials, Science 303, 2001 (2004).

[24] J. M. Cowley and A. F. Moodie, The scattering of electrons by atoms and crystals. I. A new theoretical approach, Acta Crystallogr. 10, 609 (1957).

[25] P. A. Stadelmann, EMS-A software package for electron diffraction analysis and HREM image simulation in materials science, Ultramicroscopy 21, 131 (1987). 
[26] E. J. Kirkland, Advanced Computing in Electron Microscopy, 2nd ed. (Springer New York, 2010).

[27] P. Giannozzi et al., QUANTUM ESPRESSO: A modular and open-source software project for quantum simulations of materials, J. Phys. Condens. Matter 21, 395502 (2009).

[28] J. P. Perdew, K. Burke, and M. Ernzerhof, Generalized Gradient Approximation Made Simple, Phys. Rev. Lett. 77, 3865 (1996).

[29] D. Vanderbilt, Soft self-consistent pseudopotentials in a generalized eigenvalue formalism, Phys. Rev. B 41, 7892 (1990).

[30] S. C. Jain, M. Willander, J. Narayan, and R. Van Overstraeten, III-nitrides: Growth, characterization, and properties, J. Appl. Phys. 87, 965 (2000).

[31] C.-Y. Hwang, M. J. Schurman, W. E. Mayo, Y. Li, Y. Lu, H. Liu, T. Salagaj, and R. A. Stall, Effect of substrate pretreatment on growth of $\mathrm{GaN}$ on (0001) sapphire by low pressure metalorganic chemical vapor deposition, J. Vac. Sci. Technol. A 13, 672 (1995).

[32] P. Vennéguès and B. Beaumont, Transmission electron microscopy study of the nitridation of the (0001) sapphire surface, Appl. Phys. Lett. 75, 4115 (1999).

[33] J.-L. Rouviere, C. Bougerol, B. Amstatt, E. Bellet-Almaric, and B. Daudin, Measuring local lattice polarity in AlN and GaN by high resolution Z-contrast imaging: The case of
(0001) and (100) GaN quantum dots, Appl. Phys. Lett. 92, 201904 (2008).

[34] T. Asaka, H. Banno, S. Funahashi, N. Hirosaki, and K. Fukuda, Electron density distribution and crystal structure of 27R-AlON, $\mathrm{Al}_{9} \mathrm{O}_{3} \mathrm{~N}_{7}$, J. Solid State Chem. 204, 21 (2013).

[35] T. Asaka, T. Kudo, H. Banno, S. Funahashi, N. Hirosaki, and K. Fukuda, Electron density distribution and crystal structure of 21R-AlON, $\mathrm{Al}_{7} \mathrm{O}_{3} \mathrm{~N}_{5}$, Powder Diffr. 28, 171 (2013).

[36] T. Schulz, T. Remmele, T. Markurt, M. Korytov, and M. Albrecht, Analysis of statistical compositional alloy fluctuations in $\mathrm{InGaN}$ from aberration corrected transmission electron microscopy image series, J. Appl. Phys. 112, 033106 (2012).

[37] M. Sumiya, N. Ogusu, Y. Yotsuda, M. Itoh, S. Fuke, T. Nakamura, S. Mochizuki, T. Sano, S. Kamiyama, H. Amano, and I. Akasaki, Systematic analysis and control of low-temperature GaN buffer layers on sapphire substrates, J. Appl. Phys. 93, 1311 (2003).

[38] T. I. Barry, A. T. Dinsdale, J. A. Gisby, B. Hallstedt, M. Hillert, B. Jansson, S. Jonsson, B. Sundman, and J. R. Taylor, The compound energy model for ionic solutions with applications to solid oxides, J. Phase Equilib. 13, 459 (1992). 